\title{
Multipulse operation of a Ti:sapphire laser mode locked by an ion-implanted semiconductor saturable-absorber mirror
}

\author{
M. J. Lederer and B. Luther-Davies \\ Laser Physics Centre, Research School of Physical Sciences and Engineering, The Australian National University, \\ Canberra, 0200 ACT, Australia
}

H. H. Tan and C. Jagadish

Electronic Materials Engineering, Research School of Physical Sciences and Engineering, The Australian National University, Canberra, 0200 ACT, Australia

N. N. Akhmediev

Optical Sciences Centre, Research School of Physical Sciences and Engineering, The Australian National University, Canberra, 0200 ACT, Australia

J. M. Soto-Crespo

Instituto de Optica, Consejo Superior de Investigaciones Cientificas, Serrano 121, 28006 Madrid, Spain

Received December 1, 1998; revised manuscript received February 16, 1999

\begin{abstract}
We show results obtained from a semiconductor saturable-absorber mirror mode-locked Ti:sapphire soliton laser that was operated in the multiple-pulse regime. Double, triple, and quadruple pulses were observed when the dispersion was decreased below a critical value. The pulse pairs and triplets were either widely separated or closely coupled, and spectra that resembled those of constant as well as rotating phase differences between pulses were observed. We explain our observations in the framework of the generalized complex GinzburgLandau equation as the master equation of the laser. (c) 1999 Optical Society of America [S0740-3224(99)00606-2]
\end{abstract}

OCIS codes: $140.7090,140.4050,140.3590$.

\section{INTRODUCTION}

Multiple-pulse operation of soliton lasers has been reported from time to time during the past decade. An early report of the observation of higher-order solitons is that of double-humped pulses from a colliding pulse mode-locked dye laser. ${ }^{1}$ The autocorrelation and spectral features closely resembled those of asymmetric $N$ $=2$ solitons of the nonlinear Schrödinger equation (NLSE). A large number of randomly spaced solitons were observed within the cavity of a figure-eight fiber laser. $^{2}$ In this context it was found that the intracavity energy was quantized, increasing in steps with the number of solitons present. More recently, the breakup of single pulses into multiple pulses was seen in a Kerr lens mode-locked Ti:sapphire laser ${ }^{3}$ as well as in other solidstate lasers mode locked by semiconductor saturableabsorber mirrors (SESAM's). ${ }^{4}$ In these cases the spacing between pulses was generally much larger than the single-pulse width, was irregular, and was subject to spontaneous changes.

The appearance of multiple pulses within a laser cavity is of significance to the production of high-repetition-rate soliton pulse sources for optical fiber communication sys- tems. In this context considerable effort has been directed toward refining harmonic mode locking in erbiumdoped fiber lasers ${ }^{5-9}$ as well as in a $\mathrm{Cr}^{4+}$ :YAG bulk laser. $^{10}$ In these systems either nonlinear polarization rotation or a semiconductor Bragg reflector was used as the passive mode-locking mechanism. The appearance of self-organized multiple pulses in each round trip of the cavity was attributed to a number of causes, such as coupling of the solitons by means of the dispersive continuum and acoustic waves ${ }^{5,7,11}$ or to gain dynamics. ${ }^{9,12}$ However, explanations of the conditions that lead to the formation of multiple solitons have generally been of a rather qualitative nature..$^{8,10,13}$

In this paper we are concerned with the multiple-pulse operation of a Ti:sapphire laser that is mode locked by a SESAM. In addition to widely separated double, triple, and quadruple pulses with irregular spacing, we have also found closely coupled states that are the result of interplay between saturable-absorber and filter losses, saturated gain and the coherent interaction of solitons. Emphasis lies on the explanation of these observations as well as of the mechanisms involved in the transitions from single to multiple-pulse states within the framework 
of the Ginzburg-Landau master equation used to model the laser.

\section{EXPERIMENT}

\section{A. Setup}

We performed mode-locking experiments by using ionimplanted GaAs-based SESAM's as passive mode-locking elements in a commercial Ti:sapphire laser (Coherent Mira 900D). ${ }^{14,15}$ The arrangement of the laser cavity is shown in Fig. 1. The SESAM and a focusing mirror were incorporated part way along the cavity, and the birefringent filter was placed near the output coupler. Other features of the cavity were an SF10 prism-pair dispersion compensator, allowing for a maximum negative total intracavity group-delay dispersion (GDD) of $-1100 \mathrm{fs}^{2}$ $\pm 25 \mathrm{fs}^{2}$ (i.e., $\beta_{2}=-2200 \mathrm{fs}^{2} \pm 50 \mathrm{fs}^{2}$ ); a 23 -mm-long Ti:sapphire crystal; and an output coupler (reflectivity $R_{\text {oc }}=86 \%$ at $\lambda=840 \mathrm{~nm}$ ). An $A B C D$-matrix calculation of the resonator indicated that, because of the asymmetry and astigmatism introduced by addition of the SESAM, there were two narrow and separate stability bands that corresponded to the location of mirror $\mathrm{M}_{1}$ within $<1$-mm-wide ranges; this was confirmed experimentally. The output beam for operation within one of these stability bands was elliptical, with a horizontal-tovertical beam radius ratio of $w_{x} / w_{y} \approx 1.5$; the calculated spot diameter on the SESAM was $\approx 50 \mu \mathrm{m}$. Operation in the other stability band resulted in a smaller but round output beam and a SESAM spot diameter of $\approx 120 \mu \mathrm{m}$. With a saturation fluence of $F_{\text {sat }} \approx 100 \mu \mathrm{J} / \mathrm{cm}^{2},{ }^{15}$ these SESAM spot sizes lead to saturation energies of $E_{a} \approx 2 \mathrm{~nJ}$ and of $E_{a} \approx 10 \mathrm{~nJ}$, respectively. While it was operating in either of the stability bands, the laser mode locked in a self-starting fashion for wavelengths of 820$870 \mathrm{~nm}$, with mode-locking buildup times from $3 \mathrm{~ms}$ to 30 $\mu \mathrm{s}$. Single pulses per round trip as short as $105 \mathrm{fs}$ were achieved in this configuration with the mode-locking driving force provided by the SESAM's. The SEAM's had response times of $100-500$ fs and maximum reflectivity modulations of $0.5-2 \%$, both of which depended on the conditions of the ion-implantation process as well as on the operating wavelength. Generally the modulation depths were smallest at $\lambda=820 \mathrm{~nm}$ and largest at $\lambda=870 \mathrm{~nm}$. Note that the beam-limiting aperture that is present in the standard Mira resonator was removed and that no mode-locking was observed in the modified resonator when the SESAM was replaced by a dielectric mirror.

\section{B. Observations}

Once mode locking had been established, tuning the intracavity dispersion through insertion of prism $\mathrm{P}_{2}$ resulted in consistent evolution of the pulse properties (see Fig. 2). This behavior is summarized as follows.

Starting from single-pulse operation at large negative dispersion $\left(\beta_{2}=-2000 \mathrm{fs}^{2}\right)$, decreasing $\left|\beta_{2}\right|$ led to a linear reduction in the single-pulse autocorrelation width $\tau_{\mathrm{ac}}$ from 250 to $155 \mathrm{fs}$ at $\beta_{2}=-1250 \mathrm{fs}^{2}$, as would be expected for solitonic mode locking. The reduction in dispersion was accompanied by a slight decrease in average output power, which suggests that the intracavity dy-

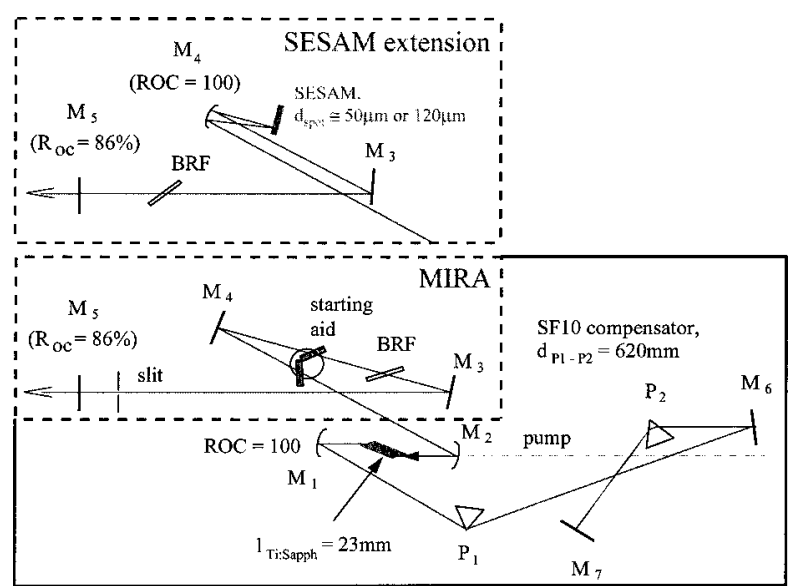

Fig. 1. Schematic of the Ti:sapphire laser cavity used in the mode-locking experiment: M's, mirrors; P's, prisms; BRF's, birefringent filters. ROC, radius of curvature.

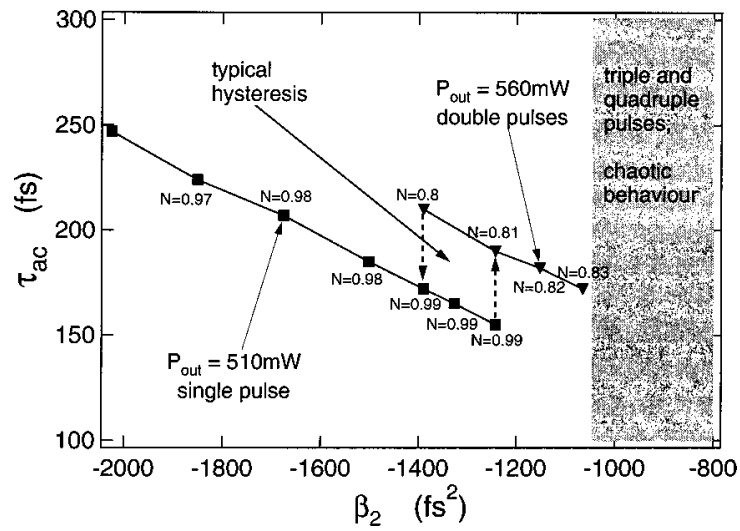

Fig. 2. Measured autocorrelation width versus total intracavity dispersion $\beta_{2}$ in the range of the single-to-double soliton transition with soliton number $N$ annotated.

namic losses increased as the dispersion was lowered. In the case of the data shown in Fig. 2, this decrease was however counteracted through slight readjustment of the pump power to keep the output power $\mathrm{P}_{\text {out }}$ constant at 510 $\mathrm{mW}$. A further decrease in dispersion below $\beta_{2}$ $=-1250 \mathrm{fs}^{2}$ led to an abrupt transition from a single pulse to double pulses circulating within the resonator. This transition was accompanied by an increase in $\mathrm{P}_{\text {out }}$ to $560 \mathrm{~mW}$, and the autocorrelation widths of the individual pulses in the double-pulse pair also increased compared with the single-pulse autocorrelation width on the high dispersion side of the transition. Similar transitions were reported previously in other SESAM and semiconductor Bragg reflector mode-locked soliton lasers. ${ }^{4,10}$ However, from the pulse energies, and assuming firstorder solitons for both the single- and the double-pulse cases, one should expect a larger jump in pulse width than that indicated in Fig. 2. We have therefore annotated the soliton number $N=\left(\kappa Q t_{0} / 2\left|\beta_{2}\right|\right)^{1 / 2}$ (Ref. 16) to the measured values of $\tau_{\text {ac }}$, assuming-sech ${ }^{2}$ pulses. Here $Q$ is the pulse energy, $\kappa$ is the self-phase modulation parameter (Section 3 ), and $t_{0}=\tau_{\mathrm{FWHM}} / 1.763$. Whereas the pulses in the single-pulse regime are virtually ideal firstorder solitons, $N$ becomes $\sim 0.8$ in the double-pulse case. 
Such a change in $N$ could indicate that the double pulses underwent nonnegligible nonlinear formation within one round trip. Alternatively, the switch from single to double pulses could have caused a slight readjustment of the mode size in the laser crystal whereby the self-phasemodulation parameter $\kappa$ was changed. We have not investigated the exact cause of the decrease in $N$ because the analyses below, in which $N=1$ is assumed, will remain qualitatively valid.

Note that in this paper we are concentrating on dispersion as the variable to induce switching among the various pulse states. However, these states could also be changed by adjustment of the pump power and hence of the small-signal gain $g_{0}$. As a result, for fixed dispersion on the single-pulse side of the transition an increase in pump power also caused the appearance of double pulses.

Once it was in double-pulse mode, we could force the laser back to single-pulse operation by increasing $\left|\beta_{2}\right|$. The transitions from single to double and from double to single generally occurred with hysteresis in $\left|\beta_{2}\right|$, indicating a form of bistability. Note, however, that the exact nature of the pulses (their separation and relative phase) in the double-pulse state near the transition could vary as the laser was cycled through the transition zone or when it was started with a $\left|\beta_{2}\right|$ value in the double-pulse region. In fact, the observed pulse-to-pulse spacings ranged sev-
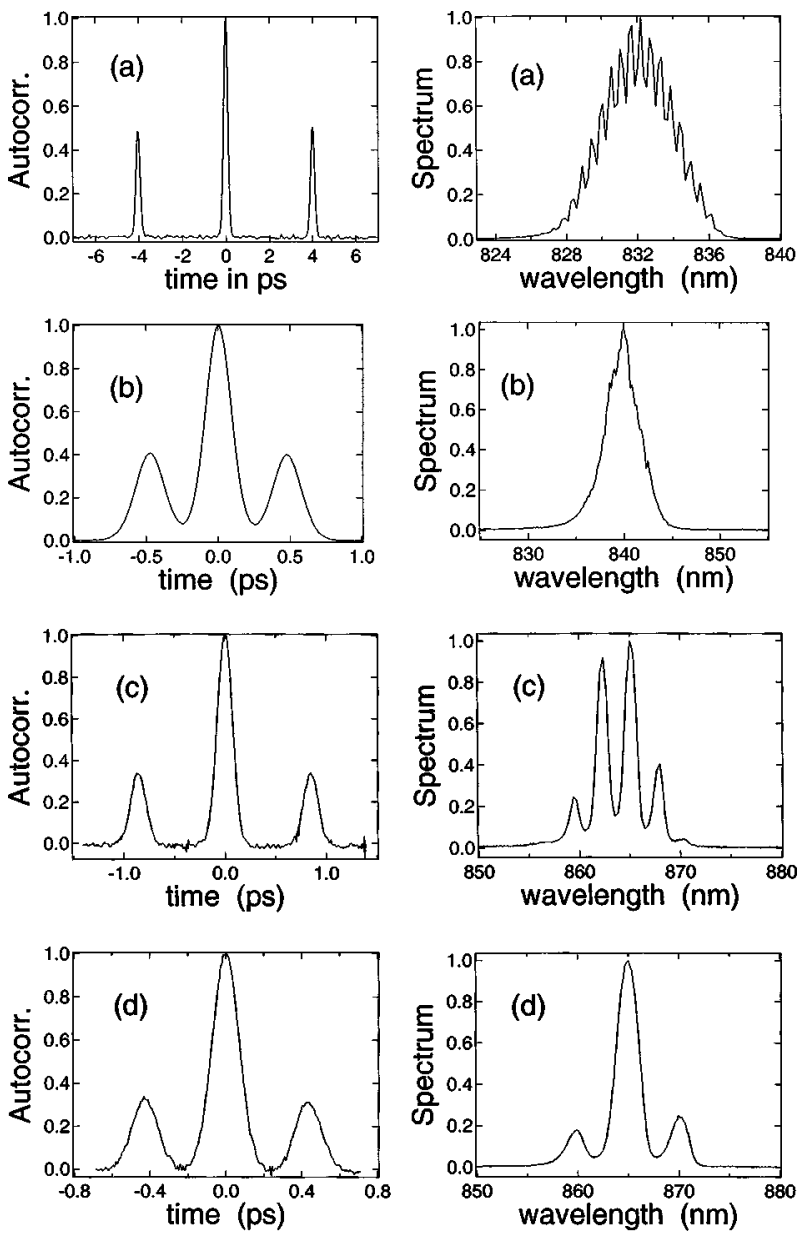

Fig. 3. Autocorrelation traces and spectra of (a) a widely separated doublet, (b) a rotating $\phi$ doublet, (c) a $\phi \approx \pi$ doublet, and (d) $\phi \approx 0$ doublet. eral hundred femtoseconds to several picoseconds, as can be seen from sample autocorrelation traces in Fig. 3. In general there was a tendency toward larger pulse spacings when the laser was operating at high pump power. The time-integrated spectra that correspond to the autocorrelation traces provide information, albeit limited, about the relative phases between the pulses in the double-pulse states. The spectra suggest that the relative phase between pulses, $\phi$, can assume a number of different states, which include fully rotating $\phi$ [Figs. 3(b)] and fixed at or alternating around $\phi \approx \pi$ [Figs. 3(c)] and $\phi \approx 0$ [Figs. 3(d)]. Spectra that correspond to either fixed or fully rotating phase states were observed for widely separated doublets, indicating that rather weakly selective processes were responsible for determining the relative phase in this case.

Starting with double pulses, a further reduction of $\left|\beta_{2}\right|$ led again to a linear decrease of $\tau_{\mathrm{ac}}$ and finally to the appearance of triple pulses at $\beta_{2}=-1050 \mathrm{fs}^{2}$. The doubleto-triple-pulse transition was again accompanied by a jump in average laser power, by an increase in the autocorrelation widths of the pulses on the low $\left|\beta_{2}\right|$ relative to the high $\left|\beta_{2}\right|$ side and by variability in the exact pulse spacing and relative phase of the pulses after the transition. The transition from triple pulses to double pulses could be induced with hysteresis by an increase of $\left|\beta_{2}\right|$. We found that in the triple-pulse state the separation of the pulses could differ widely (from several hundred femtoseconds to several nanoseconds). Autocorrelation traces and spectra of some triple-pulse states are shown in Figs. 4(a)-4(c). The spectral modulation that is due to the widely separated pulse in Figs. 4(a) is not resolved. The relative phases between triple pulses can be defined by use of the phase of the central pulse as the reference. For reasons of symmetry, in the case of the triplet in Figs. 4(b) the central pulse must be $\pi$ out of phase with its companions. We therefore call it a $\phi \approx \pi$ triplet; Figs. 4(c) show a $\phi \approx 0$ triplet. There was a clear tendency for both double and triple pulses to be closely spaced with apparently fixed phase, only when the laser was tuned at the long-wavelength end of the SESAM's range of operation, (i.e., where it exhibited the largest modulation depth) and only when it was operating with the smaller SESAM spot size, (i.e., under strong saturation). With a further reduction in $\left|\beta_{2}\right|$, quadruple-pulse states also appeared but were always separated by large distances $(>1$ ns). However, the dispersion ranges for triple and quadruple pulses were small and somewhat ill defined. Any further decrease of $\left|\beta_{2}\right|$ resulted in irregular, chaoslike behavior, indicated by unsteady autocorrelation traces [Figs. 4(d)]. Finally, crossing to positive dispersion caused the laser to emit strongly chirped picosecond pulses [Figs. 4(e)]. We noted that the transition to orderless and irregular output occurred earlier when the laser was operating with low small-signal gain $g_{0}$, where only closely spaced double pulses were seen within a narrow range of $\left|\beta_{2}\right|$.

Finally, if the laser was started at a $\left|\beta_{2}\right|$ value in the multiple-pulse region, variable final states were observed, which generally were different from those achieved when the laser was forced from single- to multiple-pulse operation via a gradual change of dispersion. Although a par- 
ticular multiple-pulse state could be stable over extended periods, the global stability of the laser in multiple-pulse mode was poor, and any slight perturbation could provoke a change of separation.

These observations provide the essential clues to identifying the important parameters that affect the appearance of multiple pulses in the laser. Clearly the transitions between pulse states display similarities, indicating that they result from the same underlying physical processes. Furthermore, the equivalent effect of changing dispersion or small-signal gain to cause a transition suggests that these two parameters must be intimately linked in any model of the process. The observation that a transition is accompanied by a distinct change in average output power indicates that a change in saturated
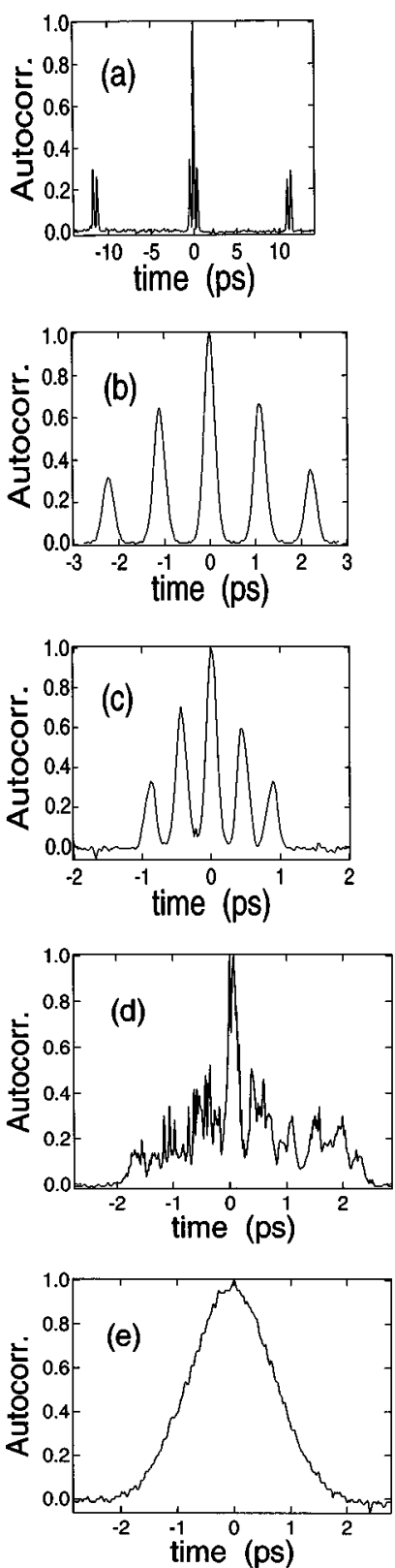

Fig. 4. Autocorrelation traces and spectra of (a) a doublet with a widely separated companion, (b) a $\phi \approx \pi$ triplet, (c) a $\phi \approx 0$ triplet, (d) irregular output for small $\left|\beta_{2}\right|$, and (e) chirped picosecond pulses for positive $\beta_{2}$. gain occurs that can be caused only by a change in the dynamic losses in the cavity. Clearly the dynamic losses also increase as $\left|\beta_{2}\right|$ is reduced in the regions of stable single or stable multiple pulses, because the average laser power decreases. Within the multiple-pulse regions, only weak processes determine the pulse separation and relative phase, because there is no strongly favored state.

Qualitatively we can link most of these factors by recognizing first that we are dealing with a soliton laser for which pulse duration and spectral bandwidth are directly related to intracavity dispersion and pulse energy. Second, it must be recognized that the intracavity dynamic losses are determined by the spectral bandwidth of the pulse through its interaction with a bandpass filter within the laser (explicitly included in our system as a birefringent tuning filter) and also by the circulating pulse power through interaction with the saturable-absorber modulator in the cavity. In explaining the observations, therefore, we focus on the relation between filter and saturable-absorber losses to the solitons within the resonator and search for regions where the laser becomes destabilized, leading to a transition to a lower loss state.

\section{THEORETICAL}

\section{A. Model}

To investigate the above observations further we used the master equation approach ${ }^{17-19}$ to describe the dynamic effects of the laser by means of numerical simulations. The relevant equation is the generalized complex Ginzburg-Landau equation ${ }^{18,19}$ (GCGLE):

$$
\begin{aligned}
i T_{R} \psi_{T}-\frac{\beta_{2}}{2} \psi_{t t}+\kappa|\psi|^{2} \psi=i\left(g-q-l_{0}\right) \psi+i \beta \psi_{t t} \\
q_{t}=\frac{q-q_{0}}{T_{a}}-\frac{|\psi|^{2} q}{E_{a}} \\
g_{T}=-\frac{g-g_{0}}{T_{g}}-\frac{Q g}{P_{g} T_{g} T_{R}}
\end{aligned}
$$

Here $T_{R}$ is the round-trip time; $\beta_{2}$ is the total intracavity dispersion; $g$ and $q$ are the saturable gain and the saturable absorption with the associated recovery times $\left(T_{g}, T_{a}\right)$, saturation energies $\left(P_{g} T_{g}, E_{a}\right)$, and unsaturated values $\left(g_{0}, q_{0}\right) ; l_{0}$ is the linear loss (output coupling, etc.); and $\beta=1 / \Omega^{2}$ describes the effect of the birefringent filter, where $\Omega$ is the HWHM of the filter. $\kappa$ is the selfphase-modulation parameter calculated from the nonlinear refractive index $n_{2}$ of sapphire, the effective mode area $A_{\text {eff }}$ inside the crystal, and the total length $l_{L}$ of the Ti:sapphire crystal per round trip, with $\kappa$ $=2 \pi n_{2} l_{L} / \lambda_{0} A_{\text {eff }}$. Further, $\psi$ represents the electricfield envelope that describes the pulses on two time scales, the soliton or retarded time $t$ and the slow time $T$ of multiple round trips. Subscripts of these time variables indicate derivatives. Eventually, $Q=\int|\psi|^{2} \mathrm{~d} t$ denotes the total intracavity energy. It is critical for the validity of this approach in conjunction with a solid-state laser with lumped elements that the fields vary by only a small amount within one round trip. We can take the ra- 
tio of round-trip time to soliton period as a measure of the strength of solitonic pulse formation ${ }^{20}$ :

$$
r=\frac{Q^{2} \kappa^{2}}{2 \pi\left|\beta_{2}\right|}=\frac{Q \kappa}{\pi t_{0}} .
$$

Therefore, if $r \ll 1$ one can expect the master equation approach to be valid. In the case of our laser, the $r$ values were of the order of 0.1 or smaller. Note that by neglecting the perturbations on the right-hand side of Eq. (1) one arrives at the well known NLSE, which has the first-order soliton solution ${ }^{16}$

$$
\psi=\sqrt{\frac{\kappa Q^{2}}{4\left|\beta_{2}\right|}} \operatorname{sech}\left(\frac{\kappa Q}{2 \times\left|\beta_{2}\right|} t\right) \exp \left(i \frac{Q^{2} \kappa^{2}}{8\left|\beta_{2}\right| T_{R}} T\right) .
$$

As an initial condition for the simulation, we either assumed that a pulse of several-picosecond width that contains all the intracavity energy and originates from the mode-coupling driving force of the saturable absorber existed in the cavity ${ }^{20}$ or used fields that were suitably close to the final solution. The parameters for the simulations are given in Table 1 and in the text when the need arises.

\section{B. Simulations}

1. Single $\leftrightarrow$ Double Transition, Relevant Mechanisms, and Widely Separated Pulses

It was pointed out in Refs. 13 and 18 that the stability of a soliton in the laser, when subject to perturbations from a filter and an absorber, can be maintained only as long as the growth of dispersive continuum radiation is effectively suppressed. This is the case when $l_{s}<l_{c}$, where

$$
l_{s}=\delta+\alpha=\frac{\beta}{3 t_{0}{ }^{2}}+\frac{1}{2 t_{0}} \int_{-\infty}^{\infty} \operatorname{sech}^{2}\left(\frac{t}{t_{0}}\right) q(t) \mathrm{d} t
$$

is the total energy loss of the soliton consisting of filter loss $\delta$ and absorber loss $\alpha$, and $l_{c}$ is the loss experienced by the continuum. For a particular $\left|\beta_{2}\right|$ the continuum loss depends mainly, in roughly inverse manner, on absorber recovery time $T_{a}$ and maximum modulation depth $q_{0}$, as was shown in Ref. 18. In the real laser, apart from filter and absorber perturbations, the discreteness of all effects (self-phase modulation, dispersion, gain, ...) causes shedding of continuum by the soliton. The discreteness

Table 1. Relevant Modeling Parameters

\begin{tabular}{lll}
\hline Parameter & \multicolumn{1}{c}{ Value } & \multicolumn{1}{c}{ Comment } \\
\hline$l_{0}$ & 0.08 & Total linear loss per round trip \\
$T_{R}$ & $12.5 \mathrm{~ns}$ & Round-trip time \\
$\Omega$ & $2 \pi \times 25 \mathrm{THz}$ & Filter HWHM \\
$\beta_{2}$ & Variable & Net intracavity dispersion \\
$\kappa$ & $1 \times 10^{-6} l / \mathrm{W}$ & Self-phase \\
& & modulation coefficient \\
$q_{0}$ & $\Delta R_{\text {max }} / 2$, & Maximum amplitude \\
$E_{a}$ & variable & modulation of SESAM \\
$T_{a}$ & Variable & Saturation energy of SESAM \\
$T_{g}$ & Variable & SESAM recovery time constant \\
$P_{g}$ & $2.5 \mu \mathrm{s}$ & Upper-state lifetime of Ti:sapphire \\
$g_{0}$ & Variable & Saturation power of Ti:sapphire \\
\hline
\end{tabular}

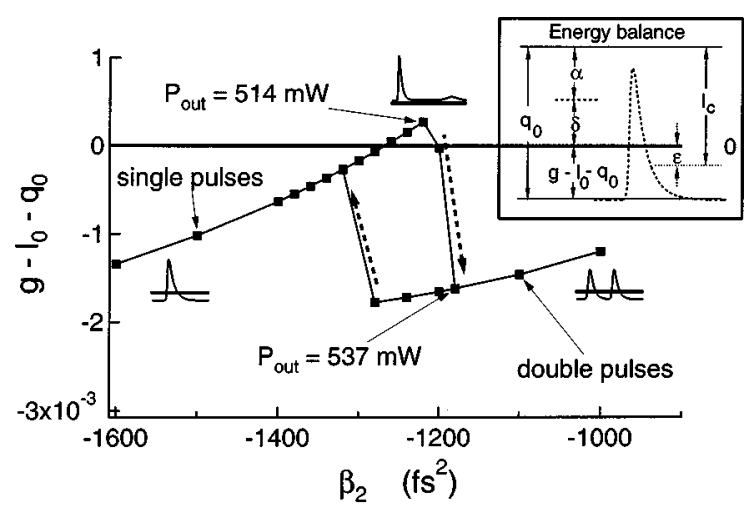

(a)

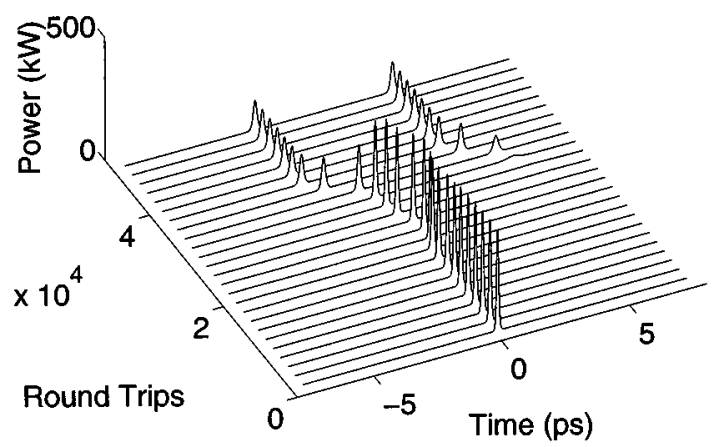

(b)

Fig. 5. (a) $g-l_{0}-q_{0}$ as a function of $\beta_{2}$ around the single-todouble transition. Inset, diagram clarifying the energy balance in equilibrium. (b) Evolution of the single soliton into two solitons with $\sim 5$-ps separation at $\beta_{2} \approx-1200 \mathrm{fs}^{2} . \quad g_{0}=0.1687$, $q_{0}=0.006, T_{a}=0.3 \mathrm{ps}, E_{a}=10 \mathrm{~nJ}, P_{g}=4 \mathrm{~W}$ (see also Table $1)$.

is not covered by the current model but will become important for cases when $r \approx 1$ or larger. Generally, for the laser in-equilibrium the condition of energy balance

$$
g-l_{0}-\delta-\alpha=0
$$

must be fulfilled, where $\delta$ and $\alpha$ are the energy losses that are due to the filter and the absorber for both solitons and continuum, if present. A graphic representation of this condition is shown in the inset of Fig. 5(a). Depending on the values of $\delta$ and $\alpha$, the laser will adjust its saturated gain $g$ such that Eq. (7) will remain fulfilled. The frame defined by the two lines separated by $q_{0}$ therefore moves with respect to the zero-gain line. Clearly, if the line $g$ $-l_{0}-q_{0}$ crosses the zero line, because of an increase in $\delta$ for instance, continuum growth is possible, even for solitonic mode-locking schemes that use ultrafast saturable absorbers such as the Kerr lens or nonlinear polarization rotation. For a slow absorber the growth occurs slightly earlier, indicated by $\epsilon \neq 0$, but the physical process is the same. Our results show that the onset of continuum growth is the critical condition that destabilizes the soliton pulse in the laser cavity. The condition for continuum growth in the presence of a single soliton with energy $Q_{1}$, and hence the threshold for the transition from single to multiple pulses, is simply

$$
\left(\alpha_{1}+\delta_{1}+\epsilon\right)_{Q_{1}} \geqslant q_{0},
$$


where $\epsilon=0$ for the ideally fast absorber. If a second stable soliton is formed at a distance $\gg T_{a}$, the two pulses have approximately half the total intracavity energy and twice the width of the single pulse before breakup; generally, this decreases $\delta$ but increases $\alpha$, which leads to the condition that

$$
\left(\alpha_{1}+\delta_{1}\right)_{Q_{1}}>\left(\alpha_{2}+\delta_{2}\right)_{Q_{2}}
$$

for the formation of two stable solitons. Here $Q_{2}$ is the total intracavity energy of two pulses, which is slightly larger (a few percent; see also Fig. 2) than $Q_{1}$. The subscripts 1 and 2 refer to the losses experienced by one and two pulses with the total energy $Q_{1}$ and $Q_{2}$, respectively. From inequality (8b) we infer that, on switching from a single to a double soliton, as long as the decrease in filter loss is greater than the increase in absorber loss the total losses are decreased, the growth of continuum is suppressed, and two stable solitons are formed. Note, however, that inequality (8a) is the breakup condition and as such is a prerequisite for inequality (8b). Equation (21) of Ref. 13 is the same as inequality (8b), although it was assigned a different significance there, namely, that of a breakup condition other than that of continuum growth. However, our numerical results show that, even if inequality (8b) is satisfied, stable single pulses exist in the resonator unless inequality (8a) is also satisfied (see e.g., Fig. 5(a), at $\beta_{2} \approx-1400 \mathrm{fs}^{2}$ ). Finally, whether the initial growth of the continuum will lead to a second soliton or will become stabilized, observable as a continuum spike on the spectrum, depends on how far $g-l_{0}-q_{0}$ has been driven above zero and therefore also on the degree of saturation and response time $T_{g}$ of the gain medium.

The stability of the pair of solitons is however, bounded by two limits. The first, toward smaller $\left|\beta_{2}\right|$, is that of renewed shedding and growth of the continuum if $\left(\alpha_{2}\right.$ $\left.+\delta_{2}+\epsilon\right)_{Q_{2}}>q_{0}$, similarly to the single-soliton case [inequalities (8)]. This process eventually drives the transition from double to triple pulses. The second limit, toward larger $\left|\beta_{2}\right|$, is not immediately obvious because, from the above argument, the pulse pair always appears to experience lower loss than a single pulse with the same total energy, because of the dominant influence of the filter loss. However, the situation becomes clearer if we derive a stability criterion from the evolution of pulse energies $Q_{a}$ and $Q_{b}$ as well as the gain $g$ in the framework of soliton perturbation theory. Here we assume that Eq. (5) remains a solution of Eq. (1) in the adiabatic sense, although the perturbations on the right-hand side are not neglected. This condition leads to

$$
\begin{aligned}
T_{R} \frac{\mathrm{d} Q_{a}}{\mathrm{~d} T} & =2\left[g\left(Q_{a}+Q_{b}\right)-l_{0}-\delta_{a}-\alpha_{a}\right] Q_{a}, \\
T_{R} \frac{\mathrm{d} Q_{b}}{\mathrm{~d} T} & =2\left[g\left(Q_{a}+Q_{b}\right)-l_{0}-\delta_{b}-\alpha_{b}\right] Q_{b}, \\
\frac{\mathrm{d} g}{\mathrm{~d} T} & =-\frac{g-g_{0}}{T_{g}}-\frac{\left(Q_{a}+Q_{b}\right) g}{P_{g} T_{g} T_{R}},
\end{aligned}
$$

where the filter and absorber losses are given by the expressions in Eq. (6) with $t_{0}=2\left|\beta_{2}\right| / \kappa Q_{a, b}$ $=\tau_{\mathrm{FWHM}} / 1.763$. From the fact that $Q_{a}=Q_{b}=Q_{\mathrm{tot}} / 2$ and $\Delta Q_{a}=-\Delta Q_{b}$ Eqs. (9)-(11) lead, after linearization, to an equation for the changes to first order in the energy of either pulse:

$$
T_{R} \frac{\mathrm{d} \Delta Q}{\mathrm{~d} T}=2 \Delta Q(\Delta g-\Delta \alpha-\Delta \delta) .
$$

Because $\Delta g=0$ initially, the condition for stability of the two solitons is therefore given by

$$
\Delta \delta>-\Delta \alpha .
$$

This means that any small perturbation of one of the solitons that leads to a change of energy and hence of pulse width can be counteracted by a change in filter loss as long as the latter is larger than the negative change in absorber loss at the current working point. If the stability condition [inequality (13)] is violated, the laser will switch back to single-pulse operation. One can expect inequality (13) to be violated when $\left|\beta_{2}\right|$ is increased, because that results in an increase in pulse duration, which reduces the absolute filter losses as well as the incremental variation in filter losses caused by the appearance of an energy imbalance. Given that the total intracavity energy always changes slightly when one switches between states $\left(Q_{1} \neq Q_{2}\right.$, owing to changes of losses and gain saturation) it is apparent that the observed hysteresis is a consequence of $Q_{1} \neq Q_{2}$ in conjunction with inequalities (8) and (13).

Figure 5(a) shows the calculated evolution of $g-l_{0}$ - $q_{0}$ as function of $\left|\beta_{2}\right|$ for the transition from one to two solitons. The parameters were chosen to match the experimental conditions of Fig. 2 as closely as possible. Starting the calculation well within the regime of singlepulse operation, we gradually changed $\left|\beta_{2}\right|$ in the simulations toward smaller values to mimic a dispersion change in the actual laser. Each gradual dispersion change was accomplished over 2000 round trips, which were then followed by 25,000 round trips of unperturbed evolution. Figure 5(a) clearly illustrates the main mechanisms involved: (a) shedding and growth of the continuum as $\left(\alpha_{1}+\delta_{1}+\epsilon\right)_{Q_{1}}>q_{0}$, (b) formation of a second soliton to reduce the total dynamic loss, moving $g-l_{0}-q_{0}$ well below the level needed for continuum growth (note that, because of the relatively fast absorber, $\epsilon \approx 0$ ), and (c) hysteresis in switching back to a single soliton as $\Delta \delta$ $<-\Delta \alpha$. Because of a lack of analytical solutions we checked the stability condition [inequality (13)] numerically for perturbations in $Q$. For the given parameters and pulse energies this condition leads to single-pulse operation for $\left|\beta_{2}\right|>1315 \mathrm{fs}^{2}$, in agreement with the simulation. Note that the single-to-double soliton transition in the simulation occurs at the same dispersion $\beta_{2}$ as in the actual laser (see Fig. 2), indicating the validity of the master equation model in this case. Figure 5(b) shows the evolution of the single pulse into two pulses with equal energies at dispersion $\beta_{2} \approx-1200 \mathrm{fs}^{2}$. Inasmuch as the continuum is suppressed by 20 orders of magnitude (not shown) and the observed separation ( $~ 5 \mathrm{ps})$ is too large for the two pulses to interact coherently or through the absorber recovery tail, they remain at a constant mean separation because of their equal energies. It must be noted that this explanation of the pulse spacing is 
valid only in the framework of the present model. It does not take into account the possibility of gain dynamics within one round trip, as considered in Ref. 12 , or acoustic effects. ${ }^{11}$ However, because of the relatively large perturbations per round trip (14\% output coupling, selfphase modulation, dispersion, and filter) in our laser, we would expect gain dynamics and acoustic effects not to be dominant in the self-organization of widely separated pulses. As we mentioned in Section 2, we did not observe reliably reproducible pulse patterns for widely separated multiple pulses and are therefore not pursuing their origins here.

\section{Closely Spaced Pulses}

It is well known that solitons of the NLSE interact, ${ }^{16} \mathrm{de}-$ pending on their relative phase and amplitude. A phase of $\phi=0$ leads to attraction and periodic collapse, whereas even small differences in amplitude and phase prevent this collapse; for $\phi=\pi$ the evolution becomes aperiodic. Owing to the similarity of the laser master equation to the NLSE we suspect that soliton-soliton interaction also plays a role in the formation of closely spaced multiple pulses of this laser. Further, if two pulses are separated by less than $\approx 5 T_{a}$, the second one will reach the absorber when it is still partly bleached, therefore incurring less absorber loss than the first pulse. In fact, the total absorber loss $\alpha_{\text {tot }}$ seen by the two pulses per round trip depends on both the separation and the relative phase between the pulses. Similarly for the filter loss, because the spectral energy of a $\phi=\pi$ pulse pair is relocated somewhat into the wings of the spectrum, where the filter loss is higher. Clearly, there are limits to both separation and phase difference of double pulses. For instance, because the laser operates in the multiplepulse regime, two pulses that attract each other owing to a zero phase difference between them cannot merge completely to form a stable single soliton, because the merged state is inherently unstable. Simulations show, however, that two pulses can merge and separate periodically with a period that is usually much longer than the soliton period for the given energy, dispersion, and self-phase modulation. Here the gain dynamics play a decisive role, reacting to the strong increase of filter loss during the merging of the pulses, when one of the pulses compresses while the other nearly disperses.

It seems more likely that stable pulse pairs exist with small separation if the relative phase is rotating such that attractive and repulsive forces average out. Additionally, the dependence of the filter and absorber losses on separation and phase of the pulses also plays a role. As a simple example, we evaluated the total loss for the case of two solitons of equal energy $E_{p}$ as a function of separation for different phases $\phi$ and absorber saturation energies $E_{a}$ (Fig. 6). We calculated Fig. 6 by neglecting the nonlinear pulse superposition that occurs for closely spaced pulses; hence the figure is valid only at separations for which the phase influence becomes negligible (at a separation of $\approx 1 \mathrm{ps}$ for the given parameters). However, it is apparent that the total loss for the two pulses increases with separation before it flattens off to the value seen by well-separated pulses. This weak potential gradient tends to favor relatively closely spaced pulses, al- though the gradient is sufficiently weak and similar for different pulse phases that there is little difference among the various possible pulse conditions. One could deduce that a range of final states could exist for identical initial conditions, as observed experimentally. Obviously if the absorber is strongly saturated, the total loss becomes dominated by the filter, and little absorber dynamics is to be expected.

We investigated the possibility of closely spaced double pulses in the GCGLE master equation [Eqs. (1)-(3)] for parameters that are realistically close to the ones that pertain to the laser. First, Fig. 7 shows the intensity autocorrelation, the time-integrated spectrum, and evolutions in the time domain as well as in the interaction plane for a pair of pulses with rotating relative phase. The interaction plane is essentially a polar plot of the pulse-to-pulse separation $\rho$ and the relative phase $\phi .{ }^{19}$ Both autocorrelation and spectrum are in good agreement with those obtained experimentally [see Figs. 3(b)] with the laser operating in either stability band. Note that the power of the first pulse is less than that of the second one, as expected from the above argument. Similarly, Figs. 8 and 9 show simulations of double pulses in which the time-integrated spectra resemble those of $\phi \approx \pi$ and

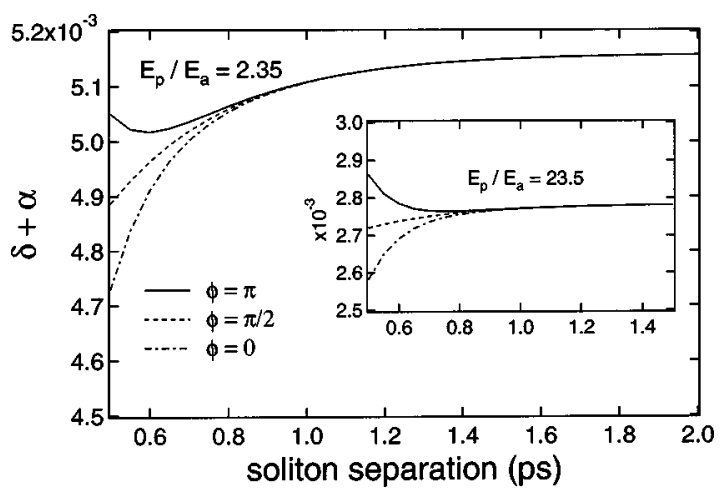

Fig. 6. Total soliton loss versus separation of two solitons of equal energy for $E_{p} / E_{a}=2.35$ and $E_{p} / E_{a}=23.5$ (inset). $\beta_{2}$ $=-900 \mathrm{fs}^{2}, T_{a}=0.3 \mathrm{ps}, E_{a}=10 \mathrm{~nJ}, q_{0}=0.006, t_{0}=77 \mathrm{fs}$ (see also Table 1).
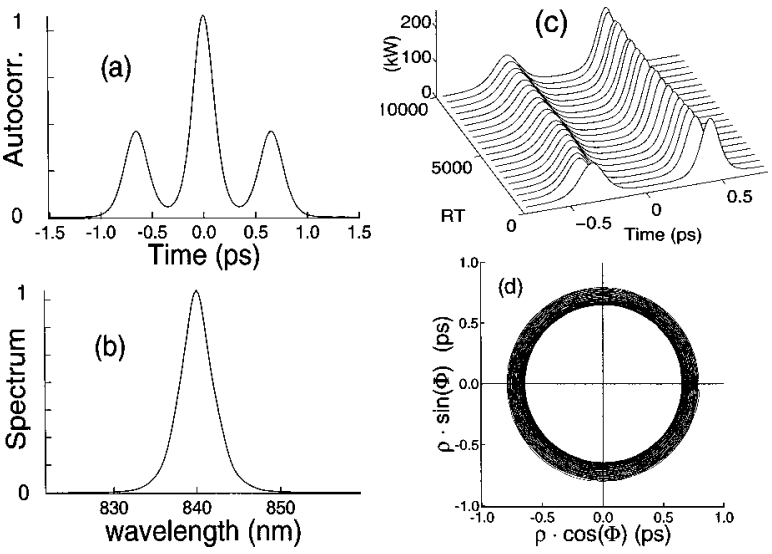

Fig. 7. Simulation results after 10,000 round trips: (a) intensity autocorrelation, (b) time-integrated spectrum, (c) evolution in the time domain, (d) interaction plane of two closely spaced pulses with rotating phase difference. $\beta_{2}=-1100 \mathrm{fs}^{2}, T_{a}$ $=0.3 \mathrm{ps}, E_{a}=10 \mathrm{~nJ}, q_{0}=0.006, g_{0}=0.2441, P_{g}=2 \mathrm{~W}$ (see also Table 1). 

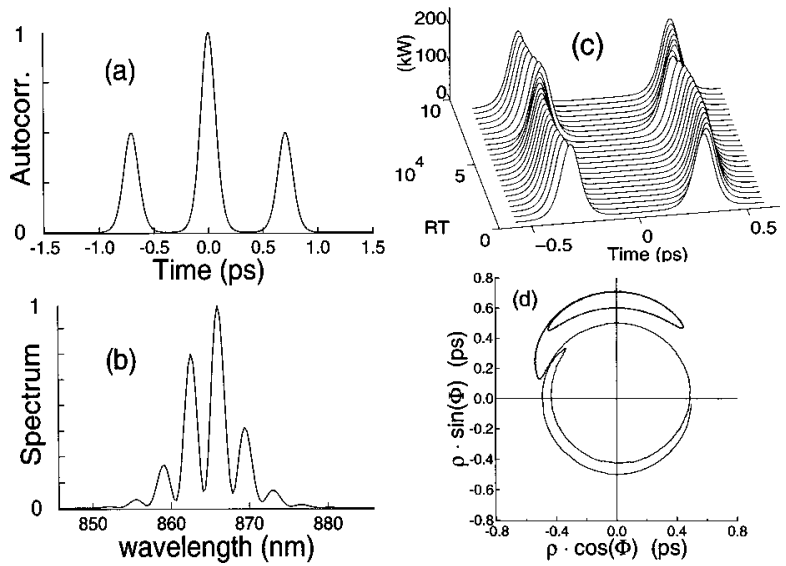

Fig. 8. Simulation results after 94,000 round trips: (a) intensity autocorrelation, (b) time-integrated spectrum, (c) evolution in the time domain, (d) interaction plane of two closely spaced pulses with mean phase difference $\phi$ between $\pi / 2$ and $\pi . \beta_{2}$ $=-688 \mathrm{fs}^{2}, T_{a}=0.1 \mathrm{ps}, E_{a}=3 \mathrm{~nJ}, q_{0}=0.01, g_{0}=0.25, P_{g}$ $=2 \mathrm{~W}$ (see also Table 1 ).
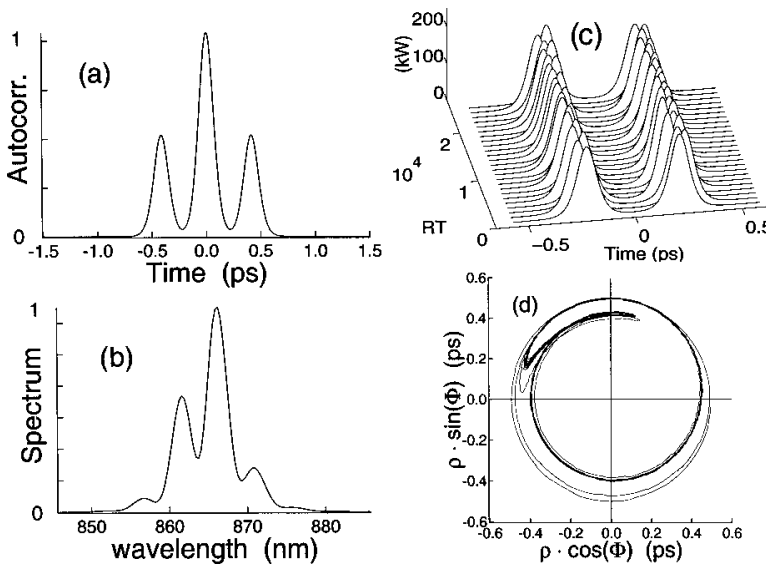

Fig. 9. Simulation results after 25,000 round trips: (a) intensity autocorrelation, (b) time-integrated spectrum, (c) evolution in the time domain, (d) interaction plane of two closely spaced pulses with mean phase difference $\phi$ between 0 and $\pi / 2$. $\beta_{2}$ $=-688 \mathrm{fs}^{2}, T_{a}=0.2 \mathrm{ps}, E_{a}=3 \mathrm{~nJ}, q_{0}=0.01, g_{0}=0.249, P_{g}$ $=2 \mathrm{~W}$ (see also Table 1 ).

$\phi \approx 0$ phase difference. Most notably, they were achieved with a strongly saturated absorber, which correlates with the fact that we observed features in the timeintegrated spectra of the experiment only for operation with the smaller SESAM spot size and hence under strong saturation. Further, as can be seen from the interaction plane, $\phi$ and $\rho$ are not constant but can evolve in complex loops with mean values of $\phi$ closer either to $\pi$ or to 0 . Finally, despite the good agreement between the simulations with the GCGLE model [Eqs. (1)-(3)] and the experiment, there are possible shortfalls as well. For instance, the SESAM differential reflectivity is only approximately described by the simple two-level rate equation (2). Generally the response is at least bitemporal, with a fast $(\approx 60-\mathrm{fs})$ transient owing to carrier thermalization and a slower one associated with carrier cooling, capturing, and recombination. Both may also depend on the excitation level because of many-body and trap filling effects. Strictly, one should also consider the nonlinear dispersive effects in the saturable absorber. Most importantly, for $r$ parameters little smaller than 1 and for strong absorber saturation the discreteness of the laser may influence closely spaced pulses differently from widely separated ones. For these cases a more refined model should be developed, with the intracavity dynamics within one round trip taken into account.

\section{Regions of Existence of Single and Multiple Pulses}

We investigated the regions of existence of single, double, and triple pulses for several values of small-signal gain $g_{0}$ and dispersion $\beta_{2}$. The laser parameters (see the caption of Fig. 10 and Table 1) were those that pertain to operation with the larger SESAM spot size, i.e., $E_{\text {sat }}$ $=10 \mathrm{~nJ}$. In a first simulation we permitted only widely separated solitons, neglecting the possibility of closely spaced pulses. Therefore we used Eqs. (9)-(11), with the number of soliton energies adjusted appropriately. Setting arbitrary initial conditions, we allowed the system to relax to the final state, where the number of solitons was noted. Figure 10(a) shows the $\left(g_{0}, \beta_{2}\right)$ plane; the single-

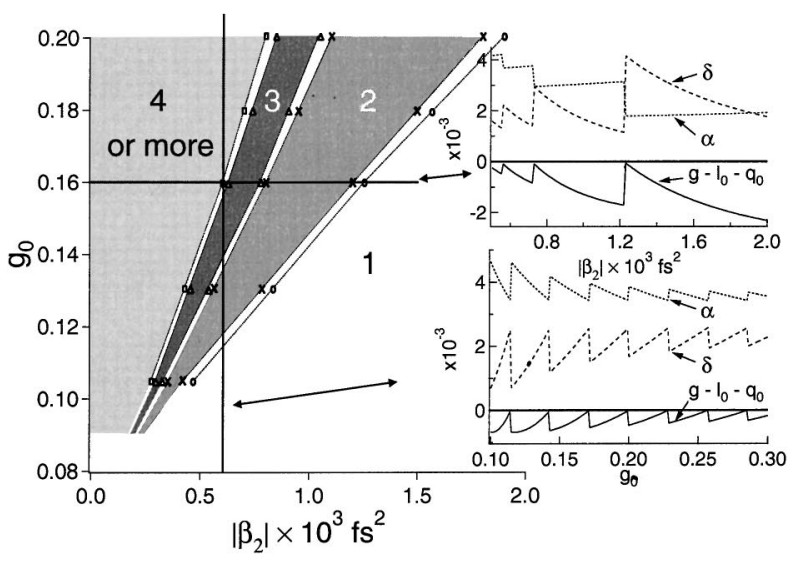

(a)

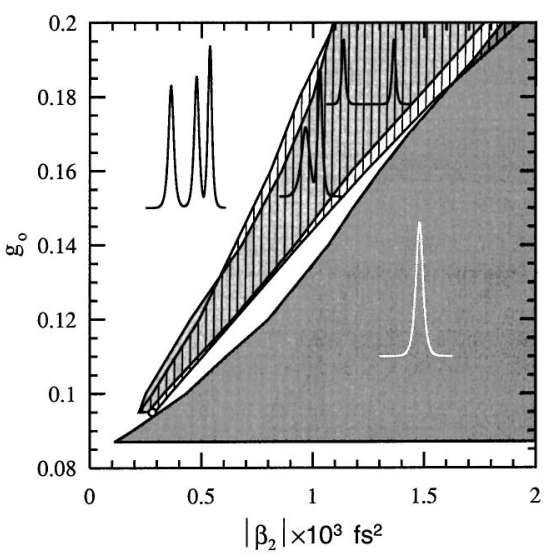

(b)

Fig. 10. (a) $\left(g_{0},\left|\beta_{2}\right|\right)$ plane showing regions of existence for single and multiple pulses according to simulations in the framework of soliton perturbation theory. Insets, $g-l_{0}-q_{0}, \delta$, and $\alpha$ for constant $g_{0}=0.16$ and $\left|\beta_{2}\right|=600 \mathrm{fs}^{2}$ respectively, as calculated from the steady-state energy balance [Eq. (7)] with $\alpha$ $+\delta>q_{0}$ as the switching condition. (b) $\left(g_{0},\left|\beta_{2}\right|\right)$ plane obtained from simulation of the complete GCGLE [Eqs. (1)-(3)]. $T_{a}=0.3 \mathrm{ps}, E_{a}=10 \mathrm{~nJ}, q_{0}=0.006, g_{0}=0.1687, P_{g}=4 \mathrm{~W}$ (see also Table 1). 
and multiple-pulse regions are indicated in different shades of gray. Within the white bands between neighboring regions the final state could fall either to the left or to the right, depending on the initial condition, indicating bistability. To underline further the sequence that leads to the appearance of multiple pulses, we depict the values of $g-l_{0}-q_{0}, \alpha$, and $\delta$ (see the insets) for constant small-signal gain $g_{0}=0.16$ and variable dispersion (indicated by the horizontal line) as well as for constant dispersion $\beta_{2}=-600 \mathrm{fs}^{2}$ and variable small-signal gain (indicated by the vertical line). Here we have simplified the simulations by solving the energy balance in steady state [Eq. (7)], using $\alpha+\delta \geqslant q_{0}$ as the switching condition. Although this treatment neglects the finite recovery time of the absorber, it emphasizes the principle that with varying dispersion or small-signal gain the laser will try to balance out the changes in the filter and absorber losses by adjusting the saturated gain such that energybalance equation (7) is fulfilled for a first-order soliton. However, once the dynamic losses become equal to the maximum possible modulation $q_{0}$ (i.e., $g-l_{0}-q_{0} \approx 0$ ), energy is transferred to another first-order soliton. The increased number of solitons renders the laser stable, because the total dynamic losses have dropped until, on a decrease in $\left|\beta_{2}\right|$ or an increase in $g_{0}$, they become equal to $q_{0}-\epsilon$ again (and so forth). For fixed $g_{0}$ and variable $\left|\beta_{2}\right|$, the width of the bands is variable, mainly because of the dependence of the absorber loss on the energy of the single solitons, which is inversely proportional to the number of solitons. Clearly, the bands would become arbitrarily narrow for $\left|\beta_{2}\right| \rightarrow 0$. On the other hand, for fixed $\left|\beta_{2}\right|$ and variable $g_{0}$, the width of the bands is constant, because the necessary energy for an increase in the number of solitons is supplied through an increase in $g_{0}$. The energy of the single solitons, and therefore the losses, remain approximately constant. However, the increasingly saturated gain for a large number of solitons provides a decreased stability margin. The insets of Fig. 10 nicely illustrate the recurring nature of the transitions between pulse states, underlining the fact, deduced from our experimental observations, that the same physical process is responsible for each transition.

A simulation of the $\left(g_{0}, \beta_{2}\right)$ plane by use of the full GCGLE [Eqs. (1)-(3)], also permitting closely spaced pulses, is shown in Fig. 10(b). However, the agreement between Figs. 10(a) and 10(b) is quite good, justifying the simple approach through soliton perturbation theory. The only major difference between Figs. 10(a) and 10(b) is that the GCGLE model allows for interaction between pulses through the phase- and distance-dependent absorber and filter losses and the soliton-soliton interaction. As was shown in Section 2, these effects can lead to the appearance of quasi-stable closely spaced double and triple pulses. If the multiple-pulse regions are narrow, or for $\left|\beta_{2}\right|$ values that correspond to a transition region, $g-l_{0}-q_{0}$ is close to $-\epsilon$. Then the interaction between closely spaced pulses does not permit even quasi-stable states, and the laser is in permanent nonequilibrium. An example of the resultant complex behavior is shown in Fig. 11(a), where the energy balance condition $g-l_{0}$ $-\alpha-\delta=0$ is seen to be permanently violated, leading to unsteady and nonperiodic pulse evolution, as can be

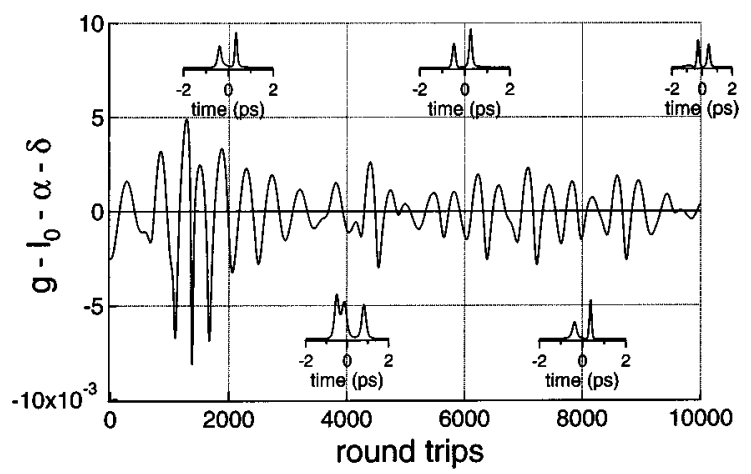

(a)

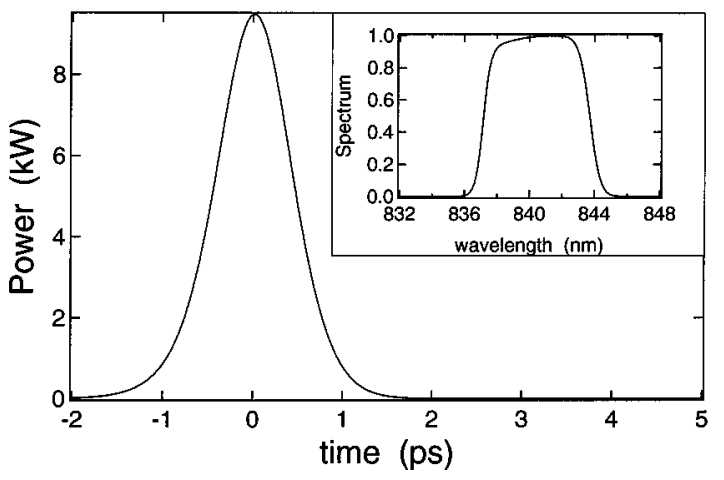

(b)

Fig. 11. (a) Simulation of the laser in nonequilibrium. The energy balance condition $g-l_{0}-\alpha-\delta=0$ is permanently violated. Also shown are the intracavity $|\psi|^{2}$ after various numbers of round trips; $\beta_{2}=-200 \mathrm{fs}^{2}$. (b) Simulation of $|\psi|^{2}$ and spectrum after 10,000 round trips for positive dispersion; $\beta_{2}$ $=+200 \mathrm{fs}^{2} . \quad T_{a}=0.3 \mathrm{ps}, \quad E_{a}=10 \mathrm{~nJ}, \quad q_{0}=0.006, \quad g_{0}$ $=0.1687, P_{g}=4 \mathrm{~W}$ (see also Table 1 ).

seen from the insets that show $|\psi|^{2}$ after various numbers of round trips. Such irregular behavior was also observed in the experiment. It is suppressed in the case of the rotating phase doublet and, to some degree, by operation of the absorber in strong saturation, as then the dynamic losses are determined mainly by the filter alone, permitting closely spaced double and triple pulses to appear. Finally, crossing over to positive dispersion makes the laser stable again; however, the laser now produces picosecond pulses, as is evident from Fig. 11(b). Since the laser is not operating in the soliton regime, the chirp that is due to self-phase modulation remains uncompensated for, and the pulses are therefore strongly chirped.

In a SESAM mode-locked soliton laser one can actually use the transition from negative to positive $\beta_{2}$ and the associated observations (single soliton $\rightarrow$ double pulses $\rightarrow$ chaoslike operation $\rightarrow$ chirped picosecond pulses) to gain a good estimate of the total intracavity $\beta_{2}$ as a function of the insertion of prism $P_{2}$. Provided that the small-signal gain $g_{0}$ is kept only as high as necessary to maintain mode locking, the transition from stable double-pulse emission to that of chirped picosecond pulses marks the crossing from negative to positive GDD and is well defined with respect to the amount of inserted $P_{2}$ glass $l_{\mathrm{P} 2-\text { glass }}$ zeroGDD $(<| \pm 0.5 \mathrm{~mm}|)$. In our experiment, this led to the quoted values for $\beta_{2}$. 


\section{CONCLUSIONS}

We have obtained solitonic mode locking from a Ti:sapphire laser, using ion-implanted SESAM's. Apart from the generation of single pulses, we have also observed that transitions between single- and multiple-pulse states can be induced by steadily reducing the value of the intracavity dispersion (or by increasing the small-signal gain). The multiple pulses could be either well separated (picoseconds...nanoseconds) without reliably reproducible spacings or closely spaced (less than picoseconds) and have their relative phase rotating or near 0 or $\pi$. The experimental observations were explained in the framework of the generalized complex Ginzburg-Landau equation.

We analyzed the single-to-double-pulse transition in detail, since it is representative of all transitions between pulse states. We found that for a single soliton to break up into two solitons the condition for shedding and growth of the dispersive continuum $\left(\alpha_{1}+\delta_{1}\right.$ $+\epsilon)_{Q_{1}} \geqslant q_{0}$ must be fulfilled. That is, when the absorber and filter losses experienced by the soliton exceed the maximum possible modulation $q_{0}$ corrected by a margin $\epsilon$ determined mainly by the recovery time of the absorber ( $\epsilon=0$ for an instantaneous recovery). On the other hand, two well-separated solitons in the laser become unstable and coalesce to form a single soliton when a change in filter loss, introduced by a slight energy imbalance, is outweighed by the simultaneous change in absorber loss $\Delta \delta<-\Delta \alpha$. We have interpreted the latter stability criterion in conjunction with the difference in total energy before and after the switching between states, $Q_{1} \neq Q_{2}$, as the origin of the observed switching hysteresis. Using computer simulations, we have further shown the existence of quasi-stable closely spaced pulse solutions of the GCGLE that originate from a balance involving soliton-soliton interactions, pulse separation, phase- and saturation-dependent absorber loss, and filter loss. The simulations were in good agreement with the experimental observations, indicating the existence of rotating phase, $\phi \approx \pi$ and $\phi \approx 0$ doublets. Finally, the regions of existence of single and multiple pulses in the $\left(g_{0}, \beta_{2}\right)$ plane were mapped through simulations. For fixed small-signal gain $g_{0}$ the regions were shown to become narrower for decreasing dispersion $\left|\beta_{2}\right|$, resulting in permanent nonequilibrium, as observed in the experiment.

\section{ACKNOWLEDGMENTS}

The authors thank A. Clark and G. Li for help with the growth and annealing of our SESAM structures and C. McLeod for expert technical support. M. J. Lederer acknowledges the support of Electro Optic Systems Pty Ltd. and the Australian Government for the award of an Australian Postgraduate Award Industry scholarship. The research of J. M. Soto-Crespo was supported by the Comunidad de Madrid under contract 06T/039/96 and by the Dirección General de Enseñanza Superior under contract PB96-0819.
M. J. Lederer's e-mail address is mj1111 @rsphysse. anu.edu.au.

\section{REFERENCES}

1. F. Salin, P. Grangier, G. Roger, and A. Brun, "Experimental observation of nonsymmetrical $N=2$ solitons in a femtosecond laser," Phys. Rev. Lett. 60, 569-572 (1988).

2. A. B. Grudinin, D. J. Richardson, and D. N. Payne, "Energy quantisation in figure eight fibre laser," Electron. Lett. 28, 67-68 (1992).

3. C. Spielmann, P. F. Curley, T. Brabec, and F. Krausz, "Ultrabroadband femtosecond lasers," IEEE J. Quantum Electron. 30, 1100-1114 (1994).

4. J. Aus der Au, D. Kopf, F. Morier-Genoud, M. Moser, and U. Keller, "60-fs pulses from a diode-pumped Nd:glass laser," Opt. Lett. 22, 307-309 (1997).

5. A. B. Grudinin, D. J. Richardson, and D. N. Payne, "Passive harmonic modelocking of a fibre soliton ring laser," Electron. Lett. 29, 1860-1861 (1993).

6. M. E. Fermann, and J. D. Minelly, "Cladding-pumped passive harmonically mode-locked fiber laser," Opt. Lett. 22, 970-972 (1996).

7. A. B. Grudinin and S. Gray, "Passive harmonic mode locking in soliton fiber lasers," J. Opt. Soc. Am. B 14, 144-154 (1997).

8. B. C. Collings, K. Bergman, S. T. Cundiff, S. Tsuda, J. N. Kutz, J. E. Cunningham, W. Y. Jan, M. Koch, and W. H. Knox, "Short cavity erbium/ytterbium fiber lasers modelocked with a saturable Bragg reflector," IEEE J. Sel. Top. Quantum Electron. 2, 1065-1074 (1997).

9. B. C. Collings, K. Bergmann, and W. H. Knox, "Stable multigigahertz pulse-train formation in a short-cavity passively harmonic mode-locked erbium/ytterbium fiber laser," Opt. Lett. 23, 123-125 (1998).

10. B. C. Collings, K. Bergmann, and W. H. Knox, "True fundamental solitons in a passively mode-locked short-cavity $\mathrm{Cr}^{4+}$ :YAG laser," Opt. Lett. 22, 1098-1100 (1997).

11. A. N. Pilipetskii, E. A. Golovchenko, and C. R. Menyuk, "Acoustic effect in passively mode-locked fiber ring lasers," Opt. Lett. 20, 907-909 (1994).

12. J. N. Kutz, B. C. Collings, K. Bergman, and W. H. Knox, "Stabilized pulse spacing in soliton lasers due to gain depletion and recovery," IEEE J. Quantum Electron. 34, 17491757 (1998).

13. F. X. Kärtner, J. Aus der Au, and U. Keller, "Mode-locking with slow and fast saturable absorbers-what's the difference?" IEEE J. Sel. Top. Quantum Electron. 4, 159-168 (1998).

14. M. J. Lederer, B. Luther-Davies, H. H. Tan, and C. Jagadish, "GaAs based antiresonant Fabry-Perot saturable absorber fabricated by metal organic vapor phase epitaxy and ion implantation," Appl. Phys. Lett. 70, 3428-3430 (1997).

15. M. J. Lederer, B. Luther-Davies, H. H. Tan, and C. Jagadish, "An anti-resonant Fabry-Perot saturable absorber for passive mode-locking fabricated by metal organic vapor phase epitaxy and ion-implantation-design, characterization and mode-locking," IEEE J. Quantum Electron. 34, 2150-2162 (1998).

16. G. P. Agrawal, Nonlinear Fiber Optics (Academic, New York, 1989).

17. H. A. Haus, "Theory of modelocking with a fast saturable absorber," J. Appl. Phys. 46, 3049-3058 (1975).

18. F. X. Kärtner, I. D. Jung, and U. Keller, "Soliton modelocking with saturable absorbers," IEEE J. Sel. Top. Quantum Electron. 2, 540-556 (1996).

19. N. Akhmediev and A. Ankiewicz, Solitons, Nonlinear Pulses and Beams (Chapman \& Hall, London, 1997).

20. F. Krausz, M. Fermann, T. Brabec, P. Curley, M. Hofer, M. Ober, C. Spielmann, E. Wintner, and A. Schmidt, "Femtosecond solid-state lasers," IEEE J. Quantum Electron. 28, 2097-2122 (1992). 